\title{
Towards an effective field theory on the light-shell
}

\author{
Howard Georgi, Greg Kestin and Aqil Sajjad \\ Center for the Fundamental Laws of Nature, \\ Jefferson Physical Laboratory, Harvard University, \\ Cambridge, MA 02138, U.S.A. \\ E-mail: georgi@physics.harvard.edu, kestin@physics.harvard.edu, \\ sajjad@physics.harvard.edu
}

ABSTRACT: We discuss our work toward the construction of a light-shell effective theory (LSET), an effective field theory for describing the matter emerging from high-energy collisions and the accompanying radiation. We work in the highly simplified venue of 0flavor scalar quantum electrodynamics, with a gauge invariant product of scalar fields at the origin of space-time as the source of high-energy charged particles. Working in this simple gauge theory allows us to focus on the essential features of LSET. We describe how the effective theory is constructed and argue that it can reproduce the full theory tree-level amplitude. We study the 1-loop radiative corrections in the LSET and suggest how the leading double-logs in the full theory at 1-loop order can be reproduced by a purely angular integral in the LSET.

KEYwords: Effective field theories, Space-Time Symmetries

ArXiv EPRINT: 1401.7667 


\section{Contents}

1 Introduction 1

2 Background 3

3 Constructing the light-shell effective theory Lagrangian 4

$\begin{array}{lll}4 & \text { LSET source } & 7\end{array}$

5 Matching LRE fields $\quad 9$

6 Reproducing full-theory results $\quad 10$

$\begin{array}{lll}6.1 \text { Tree-level } & 10\end{array}$

$\begin{array}{lll}6.2 & \text { Double logs } & 13\end{array}$

$\begin{array}{llr}7 \text { Conclusion } & 14\end{array}$

$\begin{array}{lr}\text { A 0-flavor Scalar Quantum Electrodynamics } & 15\end{array}$

$\begin{array}{ll}\text { B LRE canonical quantization } & 15\end{array}$

$\begin{array}{ll}\text { C Conventions } & 16\end{array}$

\section{Introduction}

Previously, [1] we expressed the hope of constructing an effective field theory on the 2dimensional light-shell emerging from a high-energy collision. The idea was motivated by a classical picture of a very high energy hadronic collision in which colored particles are produced from an initially color-neutral state at $t=r=0$ and instantaneously accelerate outward to the speed of light. At the same time, the collision event produces a pulse of color radiation that also moves out at the speed of light. So, classically, in the very high energy limit, everything lies on an expanding sphere at $t=r$, which we call the light-shell. It is worth mentioning that some of the recent work on assymptotic gauge symmetries has been exploring some related themes involving the null sphere at infinity [2-6].

In section 2 the classical calculation is discussed in more detail. There we observe that to have the vector potential $A^{\mu}$ confined to the light-shell requires a special gauge $-v_{\mu} A^{\mu}=0$, where $v^{\mu}$ is a light-like vector pointing away from the origin. We call this light-shell gauge (LSG).

In this paper, we begin the explicit construction of the effective field theory, incorporating the intuition gained from the classical picture by studying the quantum mechanics of particle production from a gauge invariant source at the origin of space-time. We will 
see how a gauge invariant product of scalar fields at the origin of space-time gives rise to an effective field theory of the high energy physics that depends only on the angles of the momenta of the high energy particles and fields. This 2-dimensional effective theory is our light-shell effective theory (LSET). Here we present it in the simplified venue of 0 -flavor scalar quantum electrodynamics (sQED). This strips away most of the physics so that we can focus on the construction of the LSET.

The reader might wonder why we are investing so much effort in a new effective theory so novel that we have to restrict ourselves to studying it in a toy model when there is a welldeveloped theory, SCET $[7,8]$ that is already being successfully applied to QCD processes at high energies [9]. The real answer, of course, is that we find it fascinating because our approach is very different. So, while we aim eventually to generalize our construction to QCD, even before then we hope that insight can be taken from the picture we begin to describe here. For example, an interesting feature we will discuss is that all our calculations can all be reduced to purely angular integrals.

In the classical picture, the starting point is a very high energy event in which charged particles are produced at the origin. Appropriately translating this classical setup into quantum field theory suggests a gauge invariant source at $t=r=0$. All of the physics in the effective theory will come from such a source term in our LSET Lagrangian. Since LSG is undefined at $r=0$, we construct the source on a small sphere around the origin whose size we eventually shrink to zero. In this introduction, we will also be ignoring the initial state and focusing just on the physics coming from our source. In leading order, the form of the source is fixed by gauge invariance; in section 4 we obtain the explicit form of the source.

The EFT requires that we set an energy scale $\mathcal{E}$ to define what we mean by "high energy". In the spirit of HQET [10] (for a recent and comprehensive review see [11]) we scale out the large momenta associated with the energetic outgoing particles. The associated decomposition into fields above and below $\mathcal{E}$ we call the large radial energy (LRE) expansion, and the related fields are termed LRE fields. The LRE fields correspond to high-energy particles produced by the source carrying large energy $E>\mathcal{E}$ outwards from $t=r=0$ into the bulk space. We will see that to leading order in $1 / E$, the direction $\hat{r}$ of propagation away from the origin is a classical variable and we can label the LRE fields by $\hat{r}$. But in the presence of interactions, the directions of the LRE fields cannot be specified precisely. So to each charged LRE field we assign an "angular size".

To obtain the LSET Lagrangian, we apply the LRE expansion to SQED, and expand in orders of $1 / E$. We note that the gauge interactions at leading order (in $1 / E$ ) are proportional to $v^{\mu} A_{\mu}$. So choosing LSG eliminates the gauge interactions of the LRE fields, showing that these interactions are just gauge artifacts, and that as expected, all the physics at high energies is occurring at the origin and depends only on angles. This suggests that a source at the origin is sufficient to describe the physics in LSET. Because different configurations of LRE fields (different energies and directions) do not interfere, each such configuration is associated with its own sector, and the source in the EFT is a sum over all such sectors, separated by superselection rules. 
As with any EFT, one must match to the full theory. In section 5 the LRE fields are matched to the full theory fields. Then in section 6.1 we confirm the theory's structure by showing that the source in the EFT reproduces the tree-level amplitude for the source in the full theory in the appropriate limit.

Finally, we calculate the LSET 1-loop correction for production of a scalar/anti-scalar pair. The relevant photon propagator is the LSG bulk propagator in the limit that the end points go to the light shell. We will show that for physically sensible relations between the cut-offs and $\mathcal{E}$, the effective field theory can reproduce the leading double-logs of 0 flavor sQED.

\section{Background}

LSET is motivated by a simple classical electrodynamics calculation. Consider $\mathrm{N}$ charged particles created at $\vec{r}=0$ and $t=0$, which then instantaneously accelerate to the speed of light with velocities $c \hat{r}_{j}$. The resulting electric and magnetic fields are zero everywhere in space except on an infinitesimally thin spherical shell that expands out from the origin at the speed of light. In [1], we not only motivated this idea in the context of electromagnetism but also showed that a similar picture holds for the classical non-abelian theory with the color fields associated with a non-linear $\sigma$-model on the 2D light-shell with specific symmetry breaking terms. In this paper, however, we will limit our discussion to the abelian case.

The starting point for our quantum mechanical description of this picture is a gauge invariant source at the origin of space-time that produces charged particles moving away from the origin. Gauge invariance of the source requires that the total charge vanishes,

$$
\sum_{j} q_{j}=0
$$

Because we consider a point source at the origin, our source does not conserve energy and momentum and can produce final states with any physical energy and momentum. We focus on the final states with some large energy of order $E$.

The classical observation motivates the picture of LSET, while also motivating the gauge in which we will work. If one makes an appropriate gauge transformation, not only will the electric and magnetic fields lie on a spherical shell, but the potential will as well. For the general charge configuration described above, in the appropriate gauge, our potentials are

$$
\begin{aligned}
A^{0}(t, \vec{r}) & =\sum_{j}^{N} q_{j} \delta(t-r) \log \left(t+\hat{r}_{j} \cdot \vec{r}\right) \\
\vec{A}(t, \vec{r}) & =\hat{r} \sum_{j}^{N} q_{j} \delta(t-r) \log \left(t+\hat{r}_{j} \cdot \vec{r}\right)
\end{aligned}
$$

The potentials are proportional to a $\delta$-function that sets $t=r$, so they lie on the "lightshell". An important feature is that we can write $\vec{A}(t, \vec{r})=\hat{r} A^{0}(t, \vec{r})$ satisfying the 
constraint

$$
v_{\mu} A^{\mu}=0 \quad \text { where } \quad v^{0}=1 \quad \text { and } \quad \vec{v}=\hat{r}
$$

The vector $v^{\mu}$ is a light-like vector that varies with position. (2.4) defines light-shell gauge (LSG), an essential component of the LSET effective theory.

Moving to a theory involving quantum effects, we discuss the formulation in the simplest theory, 0-flavor scalar quantum electrodynamics. In the subsequent sections, we will combine sQED with new ingredients in order to construct the the effective theory. Before doing so, we write down the important pieces of the full theory Lagrangian ${ }^{1}$ in order to refer to it later on as we mix in these new elements.

$$
\mathcal{L}_{\text {full }}=\underbrace{\left|\partial^{\mu} \phi\right|^{2}}_{\mathcal{L}_{\phi}}+\underbrace{\left(e^{2} A^{\mu} \phi A^{\mu} \phi^{*}-i e A^{\mu}\left(\left(\partial^{\mu} \phi\right) \phi^{*}+\left(\partial^{\mu} \phi^{*}\right) \phi\right)\right)}_{\mathcal{L}_{\text {int }}} \underbrace{-\frac{1}{4} F_{\mu \nu}^{2}}_{\mathcal{L}_{\mathrm{A}}}
$$

and we are interested in the functional integral

$$
\int S_{\text {full }} e^{i \int \mathcal{L}_{\text {full }} d^{4} x}[d \phi][d A] / \int e^{i \int \mathcal{L}_{\text {full }} d^{4} x}[d \phi][d A]
$$

Where $S_{\text {full }}$ is whatever gauge invariant source at the origin we wish to consider. The source describes the fields which are produced at the origin; so for example if we wish to consider the creation of a scalar/anti-scalar pair, then $S_{\text {full }}=\phi^{*}(0) \phi(0)$.

The restriction to "0-flavor" allows us to ignore matter loops and (at least at one loop) the scalar self-couplings (for details see appendix A) in our radiative corrections, which makes the physics simpler and allows us to focus narrowly on the construction of the effective field theory. Similarly, the restriction to "scalar" QED allows us to simplify the discussion of the matter in the theory. So, in theories of this kind, the only important physics is associated with the source and the radiation generated by it, and we can focus on the construction of the novel effective theory. The details of the source in LSET will be described in section 4 .

\section{Constructing the light-shell effective theory Lagrangian}

In this section we construct the terms of the LSET Lagrangian that correspond to the terms in equation (2.5), $\mathcal{L}_{\phi}, \mathcal{L}_{\text {int }}, \mathcal{L}_{A}$, and the source. As with any effective theory, the soft physics is left unchanged, so we focus on the physics associated with hard particles, and we distinguish the part of the LSET Lagrangian involving hard particles by referring to it as $\mathcal{L}_{\text {LRE }}$. This construction involves two new and essential ingredients: a field decomposition we will refer to as a large radial energy (LRE) expansion and light-shell gauge.

The large radial energy expansion is reminiscent of the field decomposition of HQET [10] and LEET (the precursor of SCET [12] that sums soft logs but not collinear logs). We scale out the uninteresting large momenta associated with the energetic particles, but as its name suggests the LRE expansion involves scaling out by a spherical wave. In

\footnotetext{
${ }^{1}$ We do not write the scalar self-interactions because they play no role in the limit we consider.
} 
order to do this, we set an energy scale $\mathcal{E}$, that determines which fields are large radial energy fields $\Phi_{E}^{(*)}$, and which fields are soft $\phi_{s}$. The decomposition is

$$
\begin{array}{r}
\phi=\phi_{s}+\sum_{E>\mathcal{E}}\left(\frac{e^{-i E(t-r)}}{\sqrt{2 E}} \Phi_{E,+q}+\frac{e^{i E(t-r)}}{\sqrt{2 E}} \Phi_{E,-q}^{*}\right) \\
\phi^{*}=\phi_{s}^{*}+\sum_{E>\mathcal{E}}\left(\frac{e^{-i E(t-r)}}{\sqrt{2 E}} \Phi_{E,-q}+\frac{e^{i E(t-r)}}{\sqrt{2 E}} \Phi_{E,+q}^{*}\right)
\end{array}
$$

where $\Phi_{E, \pm q}\left(\Phi_{E, \pm q}^{*}\right)$ annihilates (creates) high energy outgoing scalars with charge $\pm q$. In the following, we will focus on the particles with charge $+q$ and drop the $\pm q$ subscripts to simplify the tableaux. As usual in such an effective field theory decomposition, the $x$ dependence of the EFT field is assumed to be slow compared to the $t$ and $r$ dependence of the exponential factor $e^{i E(t-r)}$, and derivatives of $\Phi_{E}$ are assumed to be small compared to $\mathcal{E}$ in the effective theory. ${ }^{2}$ The $1 / \sqrt{2 E}$ is a normalization, the reason for which will soon be apparent.

Applying this expansion, the LSET Lagrangian can be written as an expansion in the small parameter $(1 / E)$, where $E$ is the energy scaled out of the energetic field at hand. Let's begin to look at $\mathcal{L}_{\mathrm{LRE}}$ by examining $\mathcal{L}_{\phi}$, the kinetic energy of our matter field, to leading order in $1 / E$. Using our expansion (3.1), focusing on the LRE terms, and using

$$
\frac{\partial(t-r)}{\partial x_{\mu}}=v^{\mu}
$$

we get

$$
\left(D^{\mu} \phi\right)^{*} D_{\mu} \phi \rightarrow \frac{1}{2 E}\left(\left(D^{\mu}+i E v^{\mu}\right) \Phi_{E}^{*}\right)\left(\left(D^{\mu}-i E v^{\mu}\right) \Phi_{E}\right)
$$

The cross terms are leading in the $1 / E$ expansion, and have a factor of $E$ from the derivatives acting on the spherical wave, which cancels the normalization from (3.1), giving

$$
=i \Phi_{E}^{*}\left(\partial_{t}+(\hat{r} \cdot \vec{\nabla}+\vec{\nabla} \cdot \hat{r}) / 2\right) \Phi_{E}+\frac{1}{2 E r^{2}} \Phi_{E}^{*} \tilde{L}^{2} \Phi_{E}
$$

where the $\tilde{L}^{2}=-r^{2}\left(\vec{\nabla}_{\perp}^{T}-i q \vec{A}_{\perp}\right) \cdot\left(\vec{\nabla}_{\perp}-i q \vec{A}_{\perp}\right)$ and we omit terms that vanish by the zeroth-order equations of motion. While the $\tilde{L}^{2}$ term is of order $1 / E$, it also has rapid $r$ dependence as $r \rightarrow 0$, which we do not want. We can make the following field redefinition to eliminate it:

$$
\tilde{\Phi}_{E}(x) \equiv \exp \left[i \frac{\tilde{L}^{2}}{2 E r}\right] \Phi_{E}(x)
$$

Note that since the derivatives in $\tilde{L}^{2}$ are all covariant, $\tilde{\Phi}_{E}(x)$ transforms just like $\Phi_{E}(x)$ under gauge transformations. In terms of $\tilde{\Phi}_{E}(x)$, and ignoring interaction terms, the kinetic energy becomes

$$
\mathcal{L}_{\phi}=i \tilde{\Phi}_{E}^{*}\left(\left(\partial_{\mu} v^{\mu}+v^{\mu} \partial_{\mu}\right) / 2\right) \tilde{\Phi}_{E}=i \tilde{\Phi}_{E}^{*}\left(\partial_{t}+(\hat{r} \cdot \vec{\nabla}+\vec{\nabla} \cdot \hat{r}) / 2\right) \tilde{\Phi}_{E}
$$

\footnotetext{
${ }^{2}$ This is a bit trickier than it sounds. See [13].
} 
The kinetic energy term (3.6) looks very much like the corresponding terms in HQET [10] and LEET [12], but there the analog of the vector $v^{\mu}$ is a constant, time-like in HQET and light-like in LEET. The fact that $v^{\mu}$ varies with $\hat{r}$ is responsible for unique properties of the LRE expansion. For example, the LRE decomposition (3.1) is invariant under rotations about the origin, not just covariant like HQET or LEET.

The $\tilde{\Phi}_{E}$ propagator associated with the kinetic energy term (3.6) is directional and has the form ${ }^{3}$

$$
\left\langle 0\left|T \tilde{\Phi}_{E}(x) \tilde{\Phi}_{E}^{*}\left(x^{\prime}\right)\right| 0\right\rangle=\frac{1}{r r^{\prime}} \theta\left(t-t^{\prime}\right) \delta\left(t-r-t^{\prime}+r^{\prime}\right) \delta\left(\hat{r}-\hat{r}^{\prime}\right)
$$

One can check (3.7) easily and it can be formally derived using canonical quantization, as we show in appendix B. The propagator (3.7) describes radially outgoing particles and this form establishes the connection between the spatial direction of the coordinate $x$ and the direction of propagation of the particle, which determines the direction of the momentum of the LRE particle far away from the source. This connection between position space and momentum space for the high-energy particles is one of the crucial components of our construction. We will return to this and see the connection very explicitly in section 5 . But while the connection is exact in the free theory, we would expect that quantum effects make it impossible to specify the momentum direction precisely. This expectation is reified in the calculation of quantum loops where specifying the directions precisely leads to divergences [14]. We assume that this is associated with the physical impossibility of measuring a jet direction exactly. Thus we associate an angular size with each LRE particle quantifying the uncertainty in direction.

We now return to equation (3.3) to explore the consequences of the LRE expansion for $\mathcal{L}_{\text {int }}$. It can be written in the suggestive form

$$
\frac{i}{2}\left[\left(-\partial^{\mu} \Phi_{E}^{\dagger}\right) v_{\mu} \Phi_{E}+v^{\mu} \Phi_{E}^{\dagger} \partial_{\mu} \Phi_{E}\right]+v_{\mu} A^{\mu} \Phi_{E}^{\dagger} \Phi_{E}+\frac{1}{2 E}\left(D^{\mu} \Phi_{E}\right)^{\dagger} D_{\mu} \Phi_{E}
$$

In this form, it is clear that in LSG our interactions vanish at leading order. The removal of the gauge interactions with LRE scalars simplifies calculations, and it makes it clear that the essential physics of the high-energy particles is associated with the source at the origin. This is consistent with the expectation of a purely angular theory on the light-shell.

Lastly, consider the purely gauge part of the Lagrangian. In LSG, $\mathcal{L}_{\mathrm{A}}$ becomes ${ }^{4}$

$$
\mathcal{L}_{A}=-\frac{1}{4} F_{\mu \nu}^{2}=-\frac{1}{2}\left(A_{r}, \vec{A}_{\perp}^{T}\right)\left(\begin{array}{cc}
\left(\partial_{t}+\vec{\nabla} \cdot \hat{r}\right)\left(\partial_{t}+\hat{r} \cdot \vec{\nabla}\right) & \left(\partial_{t}+\vec{\nabla} \cdot \hat{r}\right) \vec{\nabla}^{T} \\
\vec{\nabla}\left(\partial_{t}+\hat{r} \cdot \vec{\nabla}\right) & \vec{\nabla} \vec{\nabla}^{T}+\square I
\end{array}\right)\left(\begin{array}{c}
A_{r} \\
\overrightarrow{A_{\perp}}
\end{array}\right)
$$

Here we have used $A_{r}$ and $\vec{A}_{\perp}$, defined by

$$
\begin{aligned}
& A_{r}=\hat{r} \cdot \vec{A} \\
& \vec{A}_{\perp}=\vec{A}-\hat{r} \cdot \vec{A} \hat{r}
\end{aligned}
$$

\footnotetext{
${ }^{3}$ When $\hat{r}$ appears as an argument, it refers to dependence on angles $\theta$ and $\phi$. Likewise $\hat{r}_{j}$ refers to the angles $\theta_{j}$ and $\phi_{j}$. So, here $\delta\left(\hat{r}-\hat{r}^{\prime}\right)$ is equal to $\delta\left(z-z^{\prime}\right) \delta\left(\phi-\phi^{\prime}\right)$, with $z=\cos (\theta)$.

${ }^{4}$ This form is not completely obvious, at least to us. Details of the derivation can be found in [15].
} 
Keep in mind that in LSG the temporal component of $A^{\mu}$ is equal to $A_{r}$. An LRE expansion, similar to that of the scalars, holds for the gauge field. This expansion is in terms of longitudinal and perpendicular components of the gauge field, which is appropriate in light-shell gauge. Again, the rescaling of each field is determined by the canonical form of the kinetic energy term.

$$
\vec{A}=\vec{A}_{s}+\sum_{E>\mathcal{E}}\left(\frac{1}{\sqrt{2 E}} e^{i E(t-r)} \overrightarrow{\mathcal{A}}_{E \perp}^{*}+\frac{1}{\sqrt{2 E}} e^{-i E(t-r)} \overrightarrow{\mathcal{A}}_{E \perp}+e^{i E(t-r)} \mathcal{A}_{E r}^{*} \hat{r}+e^{-i E(t-r)} \mathcal{A}_{E r} \hat{r}+\cdots\right)
$$

After applying this expansion to (3.9) and considering the LRE terms, we can redefine the gauge field as

$$
\left(\begin{array}{l}
\mathrm{A}_{E r} \\
\overrightarrow{\mathbb{A}}_{E \perp}
\end{array}\right)=\left(\begin{array}{c}
1\left(\partial_{t}+\hat{R} \cdot \vec{\nabla}\right)^{-1} \vec{\nabla}^{T} / \sqrt{2 E} \\
0 \\
1
\end{array}\right)\left(\begin{array}{c}
A_{E r} \\
\vec{A}_{E \perp}
\end{array}\right)
$$

Where $\left(\partial_{t}+\vec{\nabla} \cdot \hat{R}\right)^{-1}$ is the inverse of a differential operator that is non-local in space and time and given explicitly by

$$
\left(\partial_{t}+\vec{\nabla} \cdot \hat{R}\right)^{-1}\left(x^{\prime}, x\right)=\frac{1}{\left(r^{\prime}\right)^{2}} \theta\left(t^{\prime}-t\right) \delta\left(t^{\prime}-r^{\prime}-t+r\right) \delta\left(\hat{r}^{\prime}-\hat{r}\right)
$$

Operators of this sort appear frequently in our LSET analysis (though we will spare the reader of this introduction most of the gory details - these will appear in [15] and [14]). These operators are treated on the same footing as linear operators, so for example, the first row of (3.13) could be written more explicitly as

$$
\mathrm{A}_{E r}(x)=A_{E r}(x)+\int\left(\left(\partial_{t}+\hat{R} \cdot \vec{\nabla}\right)^{-1}\left(x, x^{\prime}\right) \vec{\nabla}^{\prime} T / \sqrt{2 E} \vec{A}_{E \perp}\left(x^{\prime}\right) d^{4} x^{\prime}\right)
$$

In section (6.1) we will see that the quanta of the $\vec{A}_{\perp}$ field can be directly related to those of the full theory. Also, this allows us to write the LRE photon kinetic energy in the following diagonal form.

$$
\mathcal{L}_{A, \mathrm{LRE}}=\left(\begin{array}{ll}
\mathbb{A}_{E r}^{*} & \overrightarrow{\mathbb{A}}_{E \perp}^{*}
\end{array}\right)\left(\begin{array}{cc}
-\left(\partial_{t}+\vec{\nabla} \cdot \hat{r}\right)\left(\partial_{t}+\hat{r} \cdot \vec{\nabla}\right) & 0 \\
0 & \left(\partial_{t}+\hat{r} \cdot \vec{\nabla} / 2+\vec{\nabla} \cdot \hat{r} / 2\right)
\end{array}\right)\left(\begin{array}{l}
\mathbb{A}_{E r} \\
\overrightarrow{\mathbb{A}}_{E \perp}
\end{array}\right)
$$

The final piece of the LSET Lagrangian is the source. This is where the physics of our theory lies, and we describe it in the following section.

\section{LSET source}

So far we have constructed the LSET Lagrangian by bringing the large radial energy expansion and light-shell gauge to the full theory. In doing so we have removed all of the interactions of the LRE particles except for those directly associated with the point source at the origin in the full theory. The full-theory source is proportional to a gauge invariant product of local fields at the origin. Thus we also expect the corresponding source in the EFT to be gauge invariant. The conventions for the gauge transformations of our fields are listed in appendix C. 
While the full-theory source is at the origin, light-shell gauge is ill-defined there, so we begin by considering a source in the EFT that is "spread out" about the origin. We also expect from our classical picture that as the energy in the event goes to infinity, all of the physics goes onto the light shell, at $t=r$. Thus in our quantum version, we spread out our source onto a surface $r=s$ surrounding the origin, near the light-shell, with $t-r \rightarrow 0$ as $E \rightarrow \infty$. To understand the symmetry of the spread-out source, it is convenient to write $\varphi(x)=\varphi(t, r, \hat{r})$ and to let

$$
\left.\varphi(r, r, \hat{r}) \equiv \varphi(x)\right|_{t=r}
$$

represent either an LRE field or a soft field on the light shell. When we eventually write down the full source, the LRE fields will be evaluated at particular values of $\hat{r}$, while the soft fields will be integrated over $\hat{r}$. But this notation will allow us to focus on the symmetries for both types of fields simultaneously. In this notation, a term in the source spread out over $S$ appears as

$$
\mathcal{O} \propto \prod_{j} \varphi_{j}^{\dagger}\left(s, s, \hat{r}_{j}\right)
$$

This is not gauge invariant, but transforms as

$$
\mathcal{O} \rightarrow \mathcal{O} \prod_{j} \exp \left[-\left.i q_{j} \Lambda\left(x_{j}\right)\right|_{t_{j}=r_{j}=s}\right]
$$

To maintain gauge invariance we construct a compensating exponential on the light-shell

$$
\exp \left(i \frac{e}{2 \pi} \int\left(\sum_{j} \ell\left(\hat{r}, \hat{r}_{j}\right)\right) \partial_{\mu} A^{\mu}(x) d S\right)
$$

where $d S$ is our Lorentz covariant surface element on the small sphere.

$$
d S=\theta(t) r \delta(r-s) \delta\left(r^{2}-t^{2}\right) d^{4} x
$$

and assuming zero net charge

$$
\ell\left(\hat{r}, r_{j}\right)=q_{j} \log \left(1-\hat{r}_{j} \cdot \hat{r}\right)
$$

Putting all the pieces together, our gauge invariant source on the light-shell, call it $\mathcal{S}$, is of the form

$$
\begin{aligned}
C \lim _{s \rightarrow 0} \int \exp \left(i \frac{e}{2 \pi} \int\left(\sum_{j=1}^{m+n} \ell\left(\hat{r}, \hat{r}_{j}\right)\right) \partial_{\mu} A^{\mu}(x) d S\right) \\
\left(\prod_{j=1}^{m} r_{j}^{-1} \Phi_{j, E_{j}}^{\dagger}\left(x_{j}\right)\right)\left(\prod_{j=m+1}^{m+n} r_{j}^{-2} \phi_{j}^{(\dagger)}\left(x_{j}\right)\right)\left(\prod_{j=1}^{m+n} d S_{j}\right)
\end{aligned}
$$

where there are $n$ soft scalars $\phi^{(\dagger)}, m$ LRE scalars $\Phi$, and $d S_{j}$ refers to $d S$ with $x^{\mu} \rightarrow x_{j}^{\mu}$. Also, each LRE scalar $\Phi$ will have an energy associated with it $E_{i}$, this is the energy scaled out by the LRE expansion. Notice that there is a constant $C$, which must be determined by matching.

Assuming gauge transformations on the light-shell, our compensating exponential is unique. Also, one cannot help but notice the connection to the classical potential (2.3). 


\section{Matching LRE fields}

The simplest non-trivial matching to consider is that of LRE scalars. For this, we will match the amplitude of a source creating a one-particle state in the full theory to the corresponding amplitude in the effective theory. Of course, for this source to be gauge invariant, the particle must be neutral. This allows us to focus on the LRE matching all by itself. In the process, we will define creation/annihilation operators in the EFT by relating them to the familiar creation/annihilation operators in the full theory. This construction can then be carried over trivially to interesting sources involving charged particles. Let the matching condition be

$$
\langle\vec{k}| \text { Full Source }|0\rangle \stackrel{\text { match }}{=}\langle\vec{k}| \text { EFT Source }|0\rangle
$$

$\langle\vec{k}|$ is a one particle state for a scalar with momentum $k^{\mu}=(k, \vec{k})$ as defined in the full theory. This matching will connect the position space of the effective theory to the momentum space of the full theory, as well as fix the coefficient of the effective theory source. The full theory source is just $\phi(0)$. The EFT source for a high-energy particle, to leading order, has the form

$$
c_{1} \int d \Omega_{1} s\left(\hat{r}_{1}\right) \Phi_{1, E_{1}}^{\dagger}\left(s\left(\hat{r}_{1}\right), s\left(\hat{r}_{1}\right), z_{1}, \phi_{1}\right)
$$

where $c_{1}$ is the coefficient we will determine herein. The matching condition is then

$$
\langle\vec{k}|\phi(0)| 0\rangle \stackrel{\text { match }}{=}\left\langle\vec{k}\left|c_{1} \int d \Omega_{1} s\left(\hat{r}_{1}\right) \Phi_{1, E_{1}}^{\dagger}\left(s\left(\hat{r}_{1}\right), s\left(\hat{r}_{1}\right), z_{1}, \phi_{1}\right)\right| 0\right\rangle
$$

The 1.h.s. is 1 , and the r.h.s. of (5.3) is

$$
\left\langle 0\left|\sqrt{2 k} a_{k} \int d \Omega_{1} c_{1} s\left(\hat{r}_{1}\right) \Phi_{1, E_{1}}^{\dagger}\left(s\left(\hat{r}_{1}\right), s\left(\hat{r}_{1}\right), z_{1}, \phi_{1}\right)\right| 0\right\rangle
$$

Making the commutation of operators involved above well defined requires a few steps. First, define a full theory annihilation $\left(a_{s}\right)$ operator in spherical coordinates by relating it to a standard full theory operator. The familiar commutation relation is

$$
\left[a_{p}, a_{p^{\prime}}^{\dagger}\right]=(2 \pi)^{3} \delta^{(3)}\left(\vec{p}-\vec{p}^{\prime}\right)
$$

which can be expressed in spherical coordinates as

$$
\left[a_{p}, a_{p^{\prime}}^{\dagger}\right]=\frac{(2 \pi)^{3}}{p^{2}} \delta\left(p-p^{\prime}\right) \delta\left(z-z^{\prime}\right) \delta\left(\phi-\phi^{\prime}\right)
$$

The spherical creation $\left(a_{s}\right)$ and annihilation $\left(a_{s}^{\dagger}\right)$ operators we define by

$$
\left[a_{p}, a_{p^{\prime}}^{\dagger}\right] \equiv \frac{(2 \pi)^{2}}{p p^{\prime}}\left[a_{s}(p, z, \phi), a_{s}^{\dagger}\left(p^{\prime}, z^{\prime}, \phi^{\prime}\right)\right]
$$

Notice that we have

$$
\left(\frac{p}{2 \pi} a_{p}\right)\left(\frac{p^{\prime}}{2 \pi} a_{p^{\prime}}^{\dagger}\right)-\left(\frac{p^{\prime}}{2 \pi} a_{p^{\prime}}^{\dagger}\right)\left(\frac{p}{2 \pi} a_{p}\right)=a_{s}(p, z, \phi) a_{s}^{\dagger}\left(p^{\prime}, z^{\prime}, \phi^{\prime}\right)-a_{s}^{\dagger}\left(p^{\prime}, z^{\prime}, \phi^{\prime}\right) a_{s}(p, z, \phi)
$$


so the relations between conventional and spherical creation and annihilation operators are

$$
\begin{aligned}
a_{s}(p, z, \phi) & =\frac{p}{2 \pi} a_{p} \\
a_{s}^{\dagger}(p, z, \phi) & =\frac{p}{2 \pi} a_{p}^{\dagger}
\end{aligned}
$$

In the EFT we can write our fields in terms of creation/annihilation operators as

$$
\Phi^{\dagger}(t, r, \hat{r})=\int e^{i k(t-r)} \frac{1}{r} a_{\mathrm{LRE}}^{\dagger}(k, \hat{r}) \frac{d k}{2 \pi}
$$

which is described in detail in appendix B. Using the above two relations, (5.4) becomes

$$
=\int d \Omega_{1}\left\langle 0\left|\sqrt{2 k} \frac{2 \pi}{k} a_{s}(k, \hat{k}) c_{1} \int a_{\mathrm{LRE}}^{\dagger}\left(k_{1}, \hat{r}_{1}\right) \frac{d k_{1}}{2 \pi}\right| 0\right\rangle
$$

The final and crucial step is to notice that the commutation relations of $a_{s}$ and $a_{\mathrm{LRE}}$ (in appendix B) look the same, but have two important differences: $a_{s}$ involves angles in momentum space and the energy involved is the full energy $k$, whereas $a_{\mathrm{LRE}}$ involves angles in position space and the residual momentum $k$. So we identify the angles in momentum space and position space and set

$$
a_{s}^{\dagger}\left(k_{1}+k, \hat{k}\right)=a_{\mathrm{LRE}}^{\dagger}\left(k_{1}, \hat{r}\right)
$$

This allows us to turn our $a_{\mathrm{LRE}}^{\dagger}$ into $a_{s}^{\dagger}$. We find that the r.h.s. of (5.3) becomes

$$
=2 \pi \sqrt{\frac{2}{k}} c_{1}
$$

So, we have $c_{1}=\frac{1}{2 \pi} \sqrt{\frac{k}{2}}$ and our full theory scalars relate to our LRE scalars as

$$
\phi(0)=\frac{1}{2 \pi} \sqrt{\frac{k}{2}} \int d \Omega_{1} s\left(\hat{r}_{1}\right) \Phi_{1, E_{1}}^{\dagger}\left(s\left(\hat{r}_{1}\right), s\left(\hat{r}_{1}\right), \hat{r}_{1}\right)
$$

$c_{1}$ is the contribution from one LRE scalar to $\mathrm{C}$ in our general source (4.7), but we will have contributions from matching the other LRE fields involved in the process as well. While this matching procedure is fairly simple, it is essential for connecting the objects in LSET, which are formulated in position space, to the momentum-space amplitudes one is accustomed to calculating in the full theory. Relations for LRE photons operators, analogous to those introduced here, will be described in the following section.

\section{Reproducing full-theory results}

\subsection{Tree-level}

We are now prepared to compare amplitudes in the full theory and effective theory. A relevant process to compare is one with the final state of an energetic photon, scalar 
(labelled by '-'), and anti-scalar (labelled by '+'). For this comparison we will focus on the transverse component. In the full-theory, we have

$$
\left\langle\vec{k} \vec{p}_{-} \vec{p}_{+}\left|\phi^{*}(0) \phi(0)\right| 0\right\rangle=\frac{e}{|k|}\left(\frac{\hat{p}_{-}-\hat{k}\left(\hat{p}_{-} \cdot \hat{k}\right)}{1-\hat{k} \cdot \hat{p}_{-}}-\frac{\hat{p}_{+}-\hat{k}\left(\hat{p}_{+} \cdot \hat{k}\right)}{1-\hat{k} \cdot \hat{p}_{+}}\right)
$$

On the l.h.s. above, $\vec{k}$ refers to a transverse final photon state with momentum $\vec{k}$. In the effective theory we want to do the calculation with the same final state, but we now use our EFT source $\left\langle\vec{k} \vec{p}_{-} \vec{p}_{+}|\mathcal{S}| 0\right\rangle$. The dependence on scalar factors disappears. After integrating by parts and making use of the rescaling for LRE photons, we get

$$
\left\langle\vec{k}\left|\frac{-i e}{2 \pi} \int\left(\mathcal{A}_{E r}^{*}(x)\left(\partial_{t}+\hat{r} \cdot \vec{\nabla}\right)+\frac{1}{\sqrt{2 E}} \overrightarrow{\mathcal{A}}_{E \perp}^{*}(x) \cdot \vec{\nabla}\right)\left(\sum_{j=+,-} \ell\left(\hat{r}, \hat{r}_{j}\right)\right) d S\right| 0\right\rangle
$$

Note that the exponential associated with the LRE expansion has gone away because of the $\delta\left(r^{2}-t^{2}\right)$ in $d S$. Using the transformation that diagonalizes the kinetic energy (3.13) gives

$$
\begin{aligned}
= & \langle\vec{k}| \frac{-i e}{2 \pi} \int\left(\left(\delta\left(x-x^{\prime}\right) \mathbb{A}_{E r}^{*}\left(x^{\prime}\right)-\overrightarrow{\mathbb{A}}_{E \perp}^{*}\left(x^{\prime}\right) \cdot \vec{\nabla}^{\prime}\left(\partial_{t}+\vec{\nabla} \cdot \hat{R}\right)^{-1}\left(x^{\prime}, x\right) / \sqrt{2 E}\right)\left(\partial_{t}+\hat{r} \cdot \vec{\nabla}\right)\right. \\
& \left.+\delta\left(x-x^{\prime}\right) \frac{1}{\sqrt{2 E}} \overrightarrow{\mathbb{A}}_{E \perp}^{*}\left(x^{\prime}\right) \cdot \vec{\nabla}^{\prime}\right)\left(\sum_{j=+,-} \ell\left(\hat{r}, \hat{r}_{j}\right)\right) d x^{\prime} d S|0\rangle
\end{aligned}
$$

Where $\left(\partial_{t}+\vec{\nabla} \cdot \hat{R}\right)^{-1}$ is given in (3.14) and $\vec{\nabla}^{\prime}$ involves derivatives with respect to $x^{\prime}$. Since the final physical photon state is transverse, and the relevant propagator is diagonal, we can remove the term involving $\mathbb{A}_{E r}^{*}$. Then simplifying and manipulating our differential operator gives

$$
\begin{aligned}
= & \langle\vec{k}| \frac{-i e}{2 \pi} \int\left(\left(-\overrightarrow{\mathbb{A}}_{E \perp}^{*}\left(x^{\prime}\right) \cdot \vec{\nabla}^{\prime}\left(\partial_{t}+\vec{\nabla} \cdot \hat{R}\right)^{-1}\left(x^{\prime}, x\right) / \sqrt{2 E}\right)\left(\left(\partial_{t}+\vec{\nabla} \cdot \hat{r}\right)-\frac{2}{r}\right)\right. \\
& \left.+\delta\left(x-x^{\prime}\right) \frac{1}{\sqrt{2 E}} \overrightarrow{\mathbb{A}}_{E \perp}^{*}\left(x^{\prime}\right) \cdot \vec{\nabla}^{\prime}\right)\left(\sum_{j=+,-} \ell\left(\hat{r},\left\{\hat{r}_{j}\right\}\right)\right) d x^{\prime} d S|0\rangle \\
= & \left\langle\vec{k}\left|\frac{-i e}{\pi} \int\left(\overrightarrow{\mathbb{A}}_{E \perp}^{*}\left(x^{\prime}\right) \cdot \vec{\nabla}^{\prime}\left(\partial_{t}+\vec{\nabla} \cdot \hat{R}\right)^{-1}\left(x^{\prime}, x\right) \frac{1}{\sqrt{2 E} r}\right)\left(\sum_{j=+,-} \ell\left(\hat{r},\left\{\hat{r}_{j}\right\}\right)\right) d x^{\prime} d S\right| 0\right\rangle
\end{aligned}
$$

Now, as in (5.11) for scalars, can write our transverse photon field as

$$
\overrightarrow{\mathbb{A}}_{E \perp}^{*}\left(t^{\prime}, r^{\prime}, \hat{r}^{\prime}\right)=\int e^{i k\left(t^{\prime}-r^{\prime}\right)} \frac{1}{r^{\prime}} \overrightarrow{\mathrm{a}}_{E \perp}^{\dagger}\left(k, \hat{r}^{\prime}\right) \frac{d k}{2 \pi}
$$


Using this in (6.5) gives

$$
\begin{aligned}
= & \langle 0| \sqrt{2|k|} \vec{a}_{k \perp} \frac{-i e}{\pi \sqrt{2 E}} \int\left(e^{i k\left(t^{\prime}-r^{\prime}\right)} \frac{1}{r^{\prime}} \vec{a}_{E \perp}^{\dagger} \cdot \vec{\nabla}^{\prime}\left(\partial_{t}+\vec{\nabla} \cdot \hat{R}\right)^{-1}\left(x^{\prime}, x\right) \frac{1}{r}\right) \\
& \times\left(\sum_{j=+,-} \ell\left(\hat{r},\left\{\hat{r}_{j}\right\}\right)\right) \frac{d k}{2 \pi} d x^{\prime} d S|0\rangle
\end{aligned}
$$

Again, for the gauge fields' creation/annihilation operators, we can use relations analogous to those introduced in the previous section for scalars,

$$
\vec{a}_{s}(p, z, \phi)=\frac{p}{2 \pi} \vec{a}_{p}
$$

Identifying

$$
\vec{a}_{s}^{\dagger}(k+E, \hat{k})=\vec{a}_{E}^{\dagger}(k, \hat{r})
$$

allows us to have creation/annihilation operators with well-defined commutation relations. Also, note that $E=|\vec{k}|$, and (6.7) becomes

$$
\begin{aligned}
= & \langle 0| \vec{a}_{s \perp}\left(E, \hat{r}_{k}\right) \frac{-2 i e}{E} \int\left(e^{i k\left(t^{\prime}-r^{\prime}\right)} \frac{1}{r^{\prime}} \vec{a}_{s \perp}^{\dagger}\left(k+E, \hat{r}^{\prime}\right) \cdot \vec{\nabla}^{\prime}\left(\partial_{t}+\vec{\nabla} \cdot \hat{R}\right)^{-1}\left(x^{\prime}, x\right) \frac{1}{r}\right) \\
& \times\left(\sum_{j=+,-} \ell\left(\hat{r},\left\{\hat{r}_{j}\right\}\right)\right) \frac{d k}{2 \pi} d x^{\prime} d S|0\rangle
\end{aligned}
$$

The relevant commutation relation is

$$
\left[\vec{a}_{s_{\perp}}(p, z, \phi), \vec{a}_{s \perp}^{\dagger}\left(p^{\prime}, z^{\prime}, \phi^{\prime}\right)\right]=2 \pi P_{\perp} \delta\left(p-p^{\prime}\right) \delta\left(z-z^{\prime}\right) \delta\left(\phi-\phi^{\prime}\right)
$$

where $P_{\perp}$ is a projection operator for the perpendicular components. Using this we obtain

$$
=\frac{-2 i e}{E} \int\left(\delta\left(\hat{r}_{k}-\hat{r}^{\prime}\right) e^{i k\left(t^{\prime}-r^{\prime}\right)} \frac{1}{r^{\prime}} \vec{\nabla}_{\perp}^{\prime}\left(\partial_{t}+\vec{\nabla} \cdot \hat{R}\right)^{-1}\left(x^{\prime}, x\right) \frac{1}{r}\right)\left(\sum_{j=+,-} \ell\left(\hat{r},\left\{\hat{r}_{j}\right\}\right)\right) d x^{\prime} d S
$$

which involves

$$
r \vec{\nabla}_{\perp}\left(\sum_{j=+,-} \ell\left(\hat{r},\left\{\hat{r}_{j}\right\}\right)\right)=\sum_{j=+,-} q_{j}\left(\frac{\hat{r}_{j}-\hat{r}\left(\hat{r} \cdot \hat{r}_{j}\right)}{1-\hat{r} \cdot \hat{r}_{j}}\right)
$$

Using this along with integrating over $d x^{\prime}$ and $d S$ in (6.13) gives

$$
-\frac{i e}{E} \sum_{j=+,-} q_{j}\left(\frac{\hat{r}_{j}-\hat{r}_{k}\left(\hat{r}_{k} \cdot \hat{r}_{j}\right)}{1-\hat{r}_{k} \cdot \hat{r}_{j}}\right)
$$

(6.15) has the same absolute magnitude as (6.1), confirming the structure of the effective theory. 


\subsection{Double logs}

The simplest 1-loop correction to calculate is to the production of a scalar/anti-scalar pair, having energies $E_{+}$and $E_{-}$, respectively, and with $\vec{p}_{+} \cdot \vec{p}_{-}=E_{1} E_{2} z_{+-}$,

$$
\left\langle\vec{p}_{-} \vec{p}_{+}|\mathcal{S}| 0\right\rangle
$$

The pointlike source can produce any energy and momentum, so the energies, $E_{ \pm}$and directions of the scalars are unconstrained. As discussed above, the LRE expansion breaks the production process up into independent sectors, and the matrix element (6.16) picks out a particular sector in which the only hard particles produced by the source are the scalar and anti-scalar with the specified energies and angles. In the interactive theory, the radiative corrections renormalize the source in this sector to account for (for example) decreasing the amplitude for exclusive production of only charged particles with no photons. Formally, the renormalization factor is

$$
\int \exp \left(i \frac{e}{2 \pi} \int\left(\sum_{j=+,-} \ell\left(\hat{r}, \hat{r}_{j}\right)\right) \partial_{\mu} A^{\mu}(x) d S\right) e^{i \int \mathcal{L}_{\mathrm{LS}} d^{4} x}[d A] / \int e^{i \int \mathcal{L}_{\mathrm{LS}} d^{4} x}[d A]
$$

Where $\mathcal{L}_{\mathrm{LS}}$ is the gauge Lagrangian in light-shell gauge. Notice that $x$ contributes the only non-angular dependence, but $d S$ involves delta functions that leave us with purely angular dependence. This dependence solely on angles will persist for any process to any order in LSET. Evaluating (6.17) to order $e^{2}$ using the methodology introduced in [15] we arrive at

$$
=1-\frac{e^{2}}{64 \pi^{4}} \sum_{\substack{j=+,-k=+,-}} \int \frac{\left(\hat{r}_{1} \times \hat{r}_{j}\right) \cdot\left(\hat{r}_{2} \times \hat{r}_{k}\right)}{\left(1-z_{1 j}\right)\left(1-z_{12}\right)\left(1-z_{2 k}\right)} d \Omega_{1} d \Omega_{2}
$$

To obtain (6.18), we have manipulated distributions such that the result is not well-defined without regularization. For $j \neq k$, the only non-integrable singularity is at $\hat{r}_{1}=\hat{r}_{2}$. After regulating by taking $\left(1-z_{12}\right) \rightarrow\left(1-z_{12}+\lambda\right)$ we find the $j \neq k$ contribution

$$
\frac{e^{2}}{2 \pi^{2}} \log (\lambda) \log \left(1-z_{+-}\right)
$$

For $j=k$ in (6.18), the calculation is much more delicate and depends on the details of the angular cut-off around the $\hat{r}_{+}$and $\hat{r}_{-}$directions. But for physical consistency, the $\lambda$ and $\theta$ dependence must disappear as the hard emission becomes neutral. For example, if $\theta_{+} \approx \theta_{-} \approx \theta \ll 1$, the $\lambda$ dependence should cancel as $\theta_{+-} \rightarrow \theta$, because in this limit, we have two small, oppositely charged and equal-sized jets sitting right on top of one another to the level of accuracy to which we know their directions. Furthermore we expect that the $j=k$ contributions should depend on $\theta_{+}$or $\theta_{-}$, but not both, and should be symmetrical in the two. We therefore expect the leading $\log \lambda$ and $\log \theta$ dependence to have the form

$$
-\frac{e^{2}}{2 \pi^{2}} \log (\lambda) \log \left(\frac{\theta_{-} \theta_{+} / 2}{1-z_{+-}}\right)
$$

Log squared $\theta$ dependence is not possible, because it cannot be simultaneously dependent on $\theta_{+}$and $\theta_{-}$separately, symmetrical in $\theta_{+} \leftrightarrow \theta_{-}$and cancel when $\theta_{+} \approx \theta_{-} \approx \theta$. 
We now claim to have derived (6.20) from a combination of explicit calculation and consistency, but we do still wish for a better understanding of the radiative correction calculation. If we now assume (without obvious justification for now) that $\lambda$ is given by a product of the radial resolution, proportional to $\sqrt{\mathcal{E} / E_{i}}$, of the two LRE particles

$$
\lambda=\frac{\mathcal{E}}{\sqrt{E_{1} E_{2}}}
$$

then $(6.20)$ is

$$
\frac{e^{2}}{2 \pi^{2}} \log \left(\frac{\mathcal{E}}{\sqrt{E_{1} E_{2}}}\right) \log \left(\frac{\theta_{-} \theta_{+}}{1-z_{+-}}\right)
$$

This matches the double-logs from the corresponding calculation in 0-flavor sQED. This result confirms that the leading contribution from LSET can reproduce the physics of the full theory. Also, since our theory automatically disallows soft and collinear emission, this is the only double-log contribution that one should expect for the process at hand. In the full theory, one must combine the 1-loop calculation with real soft and collinear emission to achieve the same result.

\section{Conclusion}

Here we have constructed light-shell effective theory in the simplest possible case. New features essential to the construction include light-shell gauge, the large radial energy expansion, and the LSET source. After putting the pieces together, we showed that the effective theory reproduces the 0-flavor scalar quantum electrodynamics tree-level amplitude and (with appropriate choice of cut-offs) 1-loop double-logs.

We aim to extend the effective theory approach introduced here in a few ways. Our hope is to eventually reach a QCD on the light-shell. First we will need to consider the effects of fermionic matter, higher order corrections, and matter loops.

The dream of the effective field theorist is that the theory will take over - that once the appropriate degrees of freedom and symmetries are identified, everything else will fall into place. No theory for general high-energy collisions achieves this so simply. SCET's derivation, for example, takes place in a particular subset of gauges [16]. We are also not there yet with LSET since, for example, we are still using the LSG gauge propagator off the light shell as well as on. However, we find the results in this paper encouraging, and we hope they are the first steps to understanding a light-shell effective field theory for high-energy collisions.

\section{Acknowledgments}

We have benefited greatly from suggestions by Ben Grinstein, Aneesh Manohar, Matthew Schwartz, Randall Kelley, Yang Ting Chien, Ilya Feige, and David Simmons-Duffin. Some of the initial work on this project by HG was done at the Aspen Center for Physics. He is grateful for the support of the Center and National Science Foundation grant \#1066293. GK thanks the National Science Foundation Graduate Research Fellowship Program for their support. This research has also been supported at Harvard in part by the National Science Foundation under grants PHY-0804450 and and PHY-1067976. 


\section{A 0-flavor Scalar Quantum Electrodynamics}

We consider SQED with a gauge invariant source of scalars with various charges and flavor quantum numbers at the origin of space and time with a coefficient that goes to infinity as the number of flavors goes to zero so that there is a well-defined limit in which we can ignore matter loops. The details do not matter very much, but for example we could have $k$ scalar fields $\phi_{j, \alpha_{j}}$ for $j=1$ to $k$ with charges $q_{j}$ and with the flavor label $\alpha_{j}$ running from 1 to $n_{j}$ describing $n_{j}$ (identical) flavors. Our source could then look like

$$
\lambda \lim _{\{n \rightarrow 0\}} \frac{1}{K(\{n\})} \sum_{\{\alpha\}} \kappa_{\alpha_{1} \cdots \alpha_{k}} \phi_{1, \alpha_{1}}^{*} \cdots \phi_{k, \alpha_{k}}^{*}
$$

where

$$
\sum_{\{\alpha\}}\left|\kappa_{\alpha_{1} \cdots \alpha_{k}}\right|^{2}=K(\{n\})
$$

and $K(\{n\}) \rightarrow 0$ if any of the $n_{j}$ s vanish. This describes the production of $k$ "0-flavor" scalars. A trivial example is to have two fields with opposite charges and the same flavor and take $\kappa=\delta_{\alpha_{1} \alpha_{2}}$ and $K=n$. We go through this song and dance to assure the reader that we can ignore matter loops in a mathematically consistent limit without otherwise putting important restrictions on the physics. The important thing about such a source is that it produces the charged particles with charges $q_{j}$, and henceforth we will drop the flavor indices and just ignore the matter loops.

\section{B LRE canonical quantization}

The leading order Lagrangian for LRE scalars is

$$
\mathcal{L}=i \Phi_{E}^{\dagger}\left(\partial_{t}+(\hat{r} \cdot \vec{\nabla}+\vec{\nabla} \cdot \hat{r}) / 2\right) \Phi_{E}
$$

If we take $\Phi_{E}$ as the canonical field,

$$
\Pi_{E}=i \Phi_{E}^{\dagger}
$$

and we can write

$$
\Phi_{E}(t, r, \hat{r})=\int e^{-i k(t-r)} \frac{1}{r} a_{\mathrm{LRE}}(k, \hat{r}) \frac{d k}{2 \pi}
$$

with

$$
\left[a_{\mathrm{LRE}}(k, z, \phi), a_{\mathrm{LRE}}^{\dagger}\left(k^{\prime}, z^{\prime}, \phi^{\prime}\right)\right]=2 \pi \delta\left(k-k^{\prime}\right) \delta\left(z-z^{\prime}\right) \delta\left(\phi-\phi^{\prime}\right)
$$

so that the $a_{\mathrm{LRES}}$ are annihilation operators and the $a_{\mathrm{LRE}}^{\dagger} \mathrm{S}$ are creation operators. We can use the properties of the creation and annihilation operators to compute the propagator, (3.7).

Here $\Phi_{E}$ is a linear combination of annihilation operators. The positive sign of the $i$ in (B.1) is required to give the right sign of the commutation relation (B.4). This, in turn is related to having pulled out a factor of $e^{-i E(t-r)}$ from $\phi$ and a factor of $e^{i E(t-r)}$ from $\phi^{*}$, so we have built in the fact that $\Phi$ annihilates the vacuum. 
For the LRE analysis, it is crucial that $k$ is not a positive energy. It is a residual energy that can have either sign (of course it satisfies $|k| \ll \mathcal{E}$ ). Because of this, we only need the single term in (B.3) to give the canonical commutation relations. It is not possible to interpret (B.4) with only positive energies (at least, not without introducing negative-norm states).

\section{Conventions}

We use the metric

$$
g^{\mu \nu}=\left(\begin{array}{cc}
1 & 0 \\
0 & -I
\end{array}\right)
$$

Conventions for gauge transformations are

$$
\begin{gathered}
D^{\mu}=\partial^{\mu}-i e A^{\mu} \\
A^{\mu} \rightarrow A^{\mu}+\frac{1}{e} \partial^{\mu} \Lambda \\
\phi(x) \rightarrow e^{i \Lambda(x)} \phi(x)
\end{gathered}
$$

Classical calculations have been done in Heaviside-Lorentz units.

Open Access. This article is distributed under the terms of the Creative Commons Attribution License (CC-BY 4.0), which permits any use, distribution and reproduction in any medium, provided the original author(s) and source are credited.

\section{References}

[1] H. Georgi, G. Kestin and A. Sajjad, Color Fields on the Light-Shell, JHEP 02 (2016) 136 [arXiv: 1004.1404] [INSPIRE].

[2] T. He, P. Mitra and A. Strominger, 2D Kac-Moody Symmetry of $4 D$ Yang-Mills Theory, arXiv: 1503.02663 [INSPIRE].

[3] S. Pasterski, Asymptotic Symmetries and Electromagnetic Memory, arXiv:1505.00716 [INSPIRE].

[4] T. Adamo, E. Casali and D. Skinner, Perturbative gravity at null infinity, Class. Quant. Grav. 31 (2014) 225008 [arXiv:1405.5122] [INSPIRE].

[5] T. Adamo and E. Casali, Perturbative gauge theory at null infinity, Phys. Rev. D 91 (2015) 125022 [arXiv: 1504.02304] [INSPIRE].

[6] M. Campiglia and A. Laddha, Asymptotic symmetries of QED and Weinberg's soft photon theorem, JHEP 07 (2015) 115 [arXiv:1505.05346] [INSPIRE].

[7] C.W. Bauer, S. Fleming and M.E. Luke, Summing Sudakov logarithms in $\vec{B} X_{s} \gamma$ in effective field theory, Phys. Rev. D 63 (2000) 014006 [hep-ph/0005275] [INSPIRE].

[8] C.W. Bauer, S. Fleming, D. Pirjol and I.W. Stewart, An Effective field theory for collinear and soft gluons: Heavy to light decays, Phys. Rev. D 63 (2001) 114020 [hep-ph/0011336] [INSPIRE]. 
[9] M.D. Schwartz, Resummation and NLO matching of event shapes with effective field theory, Phys. Rev. D 77 (2008) 014026 [arXiv:0709.2709] [InSPIRE].

[10] H. Georgi, An Effective Field Theory for Heavy Quarks at Low-energies, Phys. Lett. B 240 (1990) 447 [inSPIRE].

[11] T. Mannel, Effective Field Theories in Flavor Physics, Springer Tracts Mod. Phys. 203 (2004) 1.

[12] M.J. Dugan and B. Grinstein, QCD basis for factorization in decays of heavy mesons, Phys. Lett. B 255 (1991) 583 [INSPIRE].

[13] M.E. Luke and A.V. Manohar, Reparametrization invariance constraints on heavy particle effective field theories, Phys. Lett. B 286 (1992) 348 [hep-ph/9205228] [INSPIRE].

[14] H. Georgi, G. Kestin and A. Sajjad, Radiative Corrections in Light-Shell Effective Theory, in preparation (2016).

[15] H. Georgi, G. Kestin and A. Sajjad, The Photon Propagator in Light-Shell Gauge, arXiv:1312.1741 [INSPIRE].

[16] I. Feige and M.D. Schwartz, An on-shell approach to factorization, Phys. Rev. D 88 (2013) 065021 [arXiv:1306.6341] [INSPIRE]. 